\title{
Trial Production of Kite Wing attached Tricopter with Tilt Front Rotors
}

\author{
Kiyoteru Hayama ${ }^{\mathrm{a}, *}$, Tomohiro Kudou ${ }^{\mathrm{b}}, \mathrm{Kazuhiro} \mathrm{Koshic}^{\mathrm{c}}$ and Hiroki Irie ${ }^{\mathrm{d}}$ \\ ${ }^{a}$ Dept. of Information, Communication and Electronic Engineering, ${ }^{b}$ Fuculty of Liberal Studies, \\ 'Dept. of Human-Oriented Information System Engineering, National Institute of Technology, Kumamoto College, \\ 2659-2 Suya, Koshi, Kumamoto 861-1102, Japan \\ ${ }^{d}$ Dept. of Architecture and Civil Engineering, National Institute of Technology, Kumamoto College, \\ 2627, Hirayamashin-machi Yatsushiro, Kumamoto, 866-0074, Japan
}

*hayama@kumamoto-nct.ac.jp

\begin{abstract}
The trial production of a new concept vertical take-off and landing (VTOL) aircraft of kite wing attached multicopter with tilt front rotos was carried out for aerial, observation and research. Continuous transition from vertical to horizontal flight can be done by tilting the front rotors. The lift of kite wing during horizontal flight supported the weight of the aircraft, and its causes the reduction of power consumption and extend the flight area.
\end{abstract}

Keywords: VTOL, kite wing, multicopter, tricopter, tilt rotor, power saving.

\section{Introduction}

The multi-rotor helicopter also called multicopter is widely spread for the less mechanical parts, electrically controllable and small space of the take-off and landing, and is useful for aerial, observation and research applications ${ }^{(1)}$. However, the time-of-flight and cruising distance is limited by the battery capacity. On the other hand, though the fixed-wing aircraft has an advantage of wide range of flight area caused by the high-speed and long-time flight of energy efficiency than the rotorcraft, it cannot hover in the air and takes a runway field to take-off and landing. Assuming the exploration and observation at disaster sites, it is difficult to secure the airfield, therefore, a new flying machine is desired that has both feature of the rotorcraft for vertical take-off and landing (VTOL) and fixed-wing aircraft for wide range of flight. There are many kind of $\mathrm{VTOL}^{(2)}$. It is well known that the tilt rotor system and tail-sitter aircraft. The tail-sitter takes off and land on its tail, then tilts horizontally for forward flight ${ }^{(3)}$. The tilt rotor system can be vertical and horizontal flight by changing the angle of rotors ${ }^{(4)}$. However, attitude control for both aircraft is difficult in transition flight to vertical and horizontal at the time of take-off and landing.

In our previous research, trial production of variable pitch wing attached multicopter and flexible kite wing attached multicopter were carried out. In both cases, it was confirmed that the lift of the wing during level flight was effective to reduce the power consumption ${ }^{(5,6)}$.

In this study, we propose a new concept VTOL aircraft of kite wing attached multicopter with tilt front rotors to earn the advantage for energy saving of wing. We aim for highly efficient flight with a complete conversion to an airplane.

\section{Proposed Rotorcraft}

\subsection{Comparison with our previous studies}

Figure 1 shows trial production of a new concept VTOL based on variable pitch wing attached multicopter ${ }^{(5)}$. Continuous transition from rotorcraft to like fixed-wing aircraft could be done with adjusting suitable angle of attack of the wing of the rotorcraft. It was confirmed that the lift of the wing during horizontal flight was effective to reduce the power consumption less than $66 \%$ compared with that without wing, and the flight area could be extended widely. However, disadvantages of the rotorcraft are also derived. The rotorcraft is difficult to climb and descend rapidly. Lift of the wing cannot be obtained except for forward flight. It is investigated the main wing is affected by side wind and turbulence generated by own rotors, which is negative factor in stability during take-off and landing of the rotorcraft. The rotorcraft has poor 
portability because dividing the main wing has a limitation for the aspect of securing its strength, in contrast to the multicopter can improve portability by folding the rod mounting rotors.

The flexible kite wing attached multicopter shown in Fig. 2 was also developed to earn the advantage of the attachment of variable pitch wing ${ }^{(6)}$. The kite wing is designed to open and close in conjunction with the change of the mounting angle. The flexible wing can be solved portability of the wing and avoiding the bad influence of turbulence by rotors during take-off and landing. It was confirmed that the lift of the kite wing during horizontal flight can be reduced the power consumption less than $79 \%$ compared with that without wing. However, Since the lift-to-drag ratio of the kite is not large and the power saving effect is small. Therefore, complete conversion to airplane is desirable.

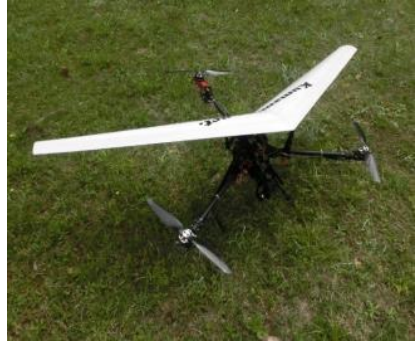

Fig. 1. Y3 tricopter with variable pitch wing.

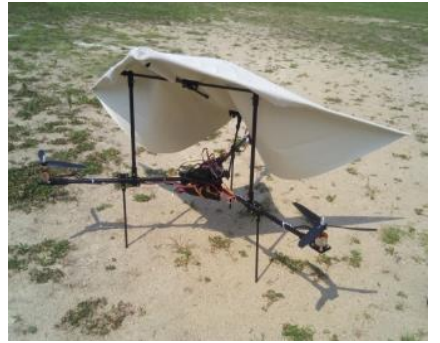

Fig. 2. Flexible kite wing attached tricopter.

\subsection{Principle of the proposed aircraft}

Figure 3 shows our new concept VTOL aircraft of kite wing attached multicopter with tilt front rotors. We aim for highly efficient flight with a complete conversion to an airplane.

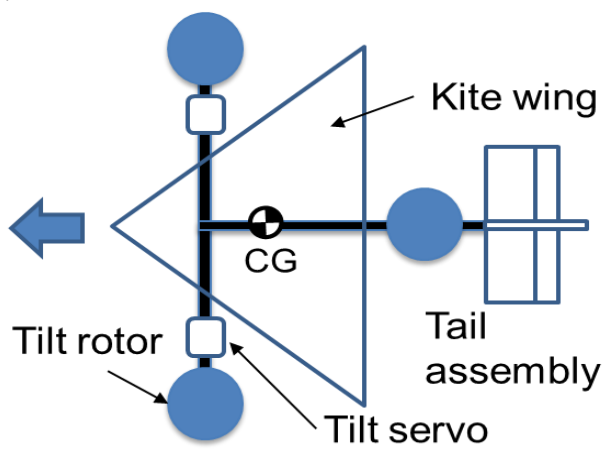

Fig. 3. Concept of the proposed aircraft

We have designed the aircraft based on tricopter which is consists of the minimum number of three rotors required for floating and attitude stabilization. Previous work has shown that kite wing has much tolerance for the pitch angle. the wing area and mounting angle are pre-fixed. Two front rotors can be tilted $90^{\circ}$ to forward by each servomotor. The T-shaped frame is used so that the rotor turned toward front when tilting. The triangular kite wing with its apex at the front has an arrangement with few interferences with the rotors. The aerodynamic center of the kite wing is designed to coincide with the center of gravity.

Figure 4 shows the weight and balance acting on the aircraft during flight. During hovering, the flight controller (FC) adjusts the thrust of three rotors to keep the balance of the aircraft as shown in fig.4(a). When flight forward, the two front rotors tilt forward (fig. 4(b)). As it is a high-wing aircraft with a large mounting angle of kite wing, the thrust force of front rotors $\left(F_{R}\right.$ and $\left.F_{L}\right)$ causes a tendency of nose up, but the FC keeps the aircraft level by decreasing the $F_{R}$ and $F_{L}$. By tilting rotors gently, the aircraft can smoothly transition to level flight. A normal multicopter flies horizontally by taking a forward attitude by changing the thrust of rotors, but the proposed aircraft can fly forward by tilting front rotors while keeping the level of airframe. When level flight, the lift of wing (L) and weight of the aircraft $(\mathrm{W})$ are balanced, also the thrust of front rotors $\left(F_{R}\right.$ and $F_{L}$ ) and drag (D) are balanced. So, the tail rotor becomes unnecessary and further power saving can be expected.

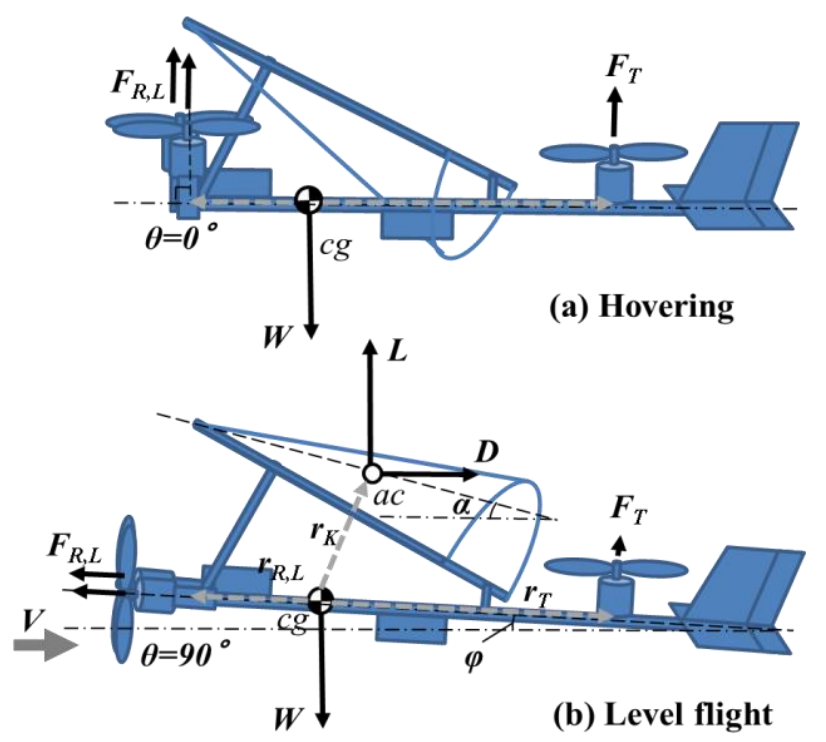

Fig. 4. Weight and balance acting on the aircraft.

\section{Trial production of the proposed aircraft}

Figure 5 shows trial production of the proposed aircraft. The base flame is T-shape tricopter, which is made by carbon rods mounted three blushless motors. The maximum thrust force and power consumption of the motor using 3 
blade 9 inches propeller are $0.56 \mathrm{~kg}$ and $180 \mathrm{~W}$ each, respectively. There is enough thrust to lift the aircraft with three rotors. The total weight including LiPo battery (3S-11.1V, 2200mAh) was about $0.96 \mathrm{~kg}$. The weight of the kite wing is $0.20 \mathrm{~kg}$. The wing area are variable ranging from 0.36 to $0.43 \mathrm{~m}^{2}$ and the mounting angle is also pre-settable.

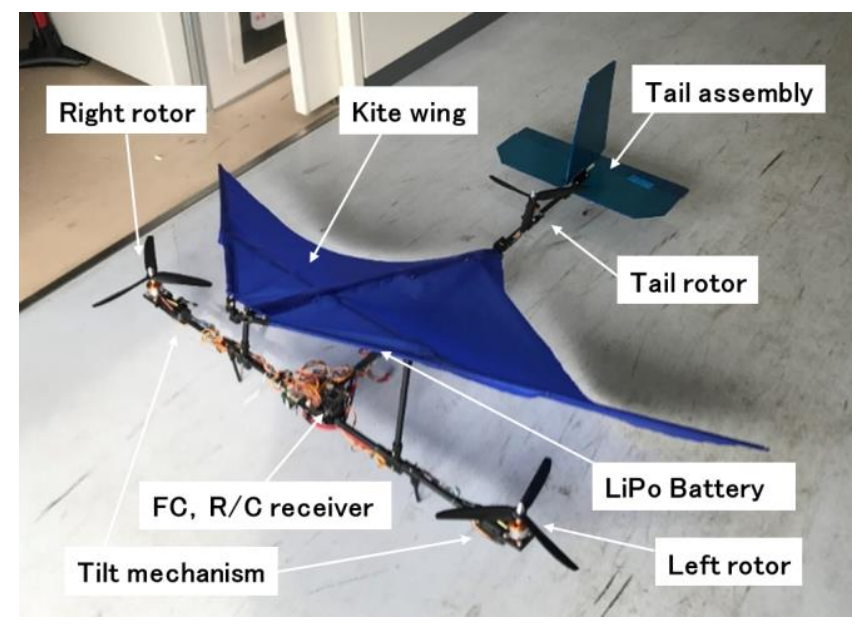

Fig. 5. Trial product of the proposed aircraft.

Figure 6 shows the block diagram of the proposed aircraft. The aircraft is operated by $2.4 \mathrm{GHz} \mathrm{R} / \mathrm{C}$ system. We have used the CC3D ${ }^{(7)}$ for attitude control of the aircraft. The CC3D is commercially available flight controller. The control board is developed as open source/hardware project. The 3-axis acceleration sensor and 3-axis gyroscope are mounted in CC3D. The configuration of the attitude and flight control are possible by using a LibrePilot ${ }^{(8)}$. The CC3D which is installed general tricopter firmware. Stabilization of both hovering and horizontal flight of the proposed aircraft is carried out by CC3D.

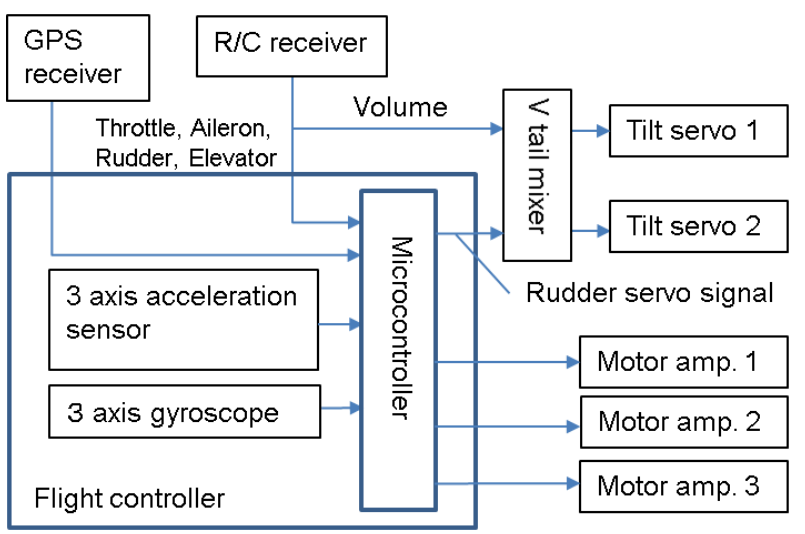

Fig. 6. Block diagram of the proposed aircraft.
Tilting of the front rotors is manually performed by volume control of the transmitter. The volume signal and the rudder servo signal from $\mathrm{FC}$ are mixed and connected to the tilt servomotors. At the same time, the PID control parameters of FC for stabilization of the airframe are changed optimally according to the tilt angle of rotors.

Figure 7 shows the tilt of the two front rotors. When the aircraft rotates on the yaw axis by rudder operation, the left and right rotors are tilted in the opposite direction as shown in Fig. 6(a). When level flight, both left and right rotors are tilted forward as shown in Fig. 6(b).

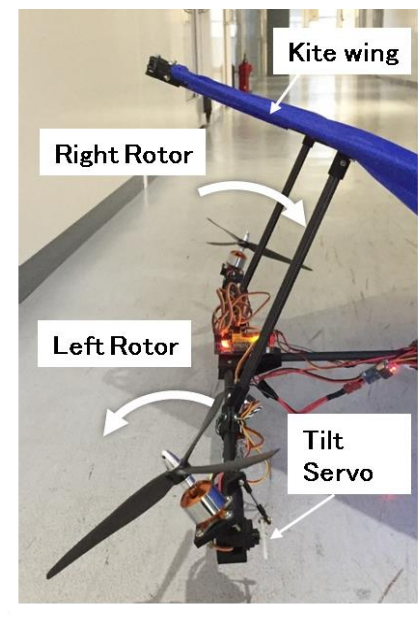

(a) Rotor tilting opposite direction

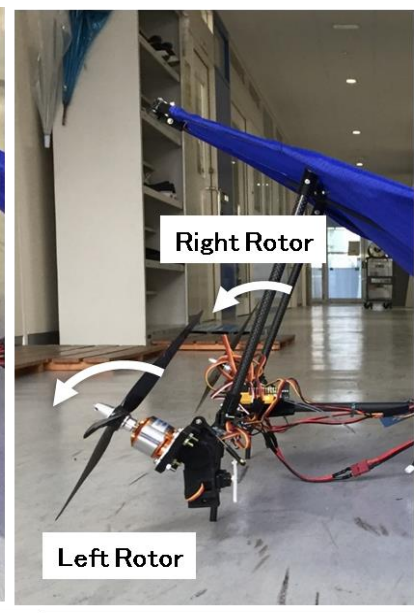

(b) Both rotor tilting forward
Fig. 7. Tilt of the front rotors.

\section{Test flight}

We conducted a test flight of the aircraft. After take-off vertically, the aircraft kept level and stable hovering was possible as shown in Fig. 8(a). The sail did not flutter during hovering and there was little influence of the air flow of the rotors.

The front rotors tilt according to the volume operation, and the aircraft smoothly transitions from hovering to level flight. Fig. 8(b) shows a photograph when the aircraft transited to level flight by tilted front rotors. Operability was also gradually changed from multicopter to airplane. After tilted the rotor, a turning flight was possible by output difference between the left and right rotors according to aileron operation. The tensioned sail is bellying in the wind. As the airspeed increases, the aircraft rises the altitude by the lift of the kite wing. In order to keep the altitude, the throttle should to be down resulting in power saving. 

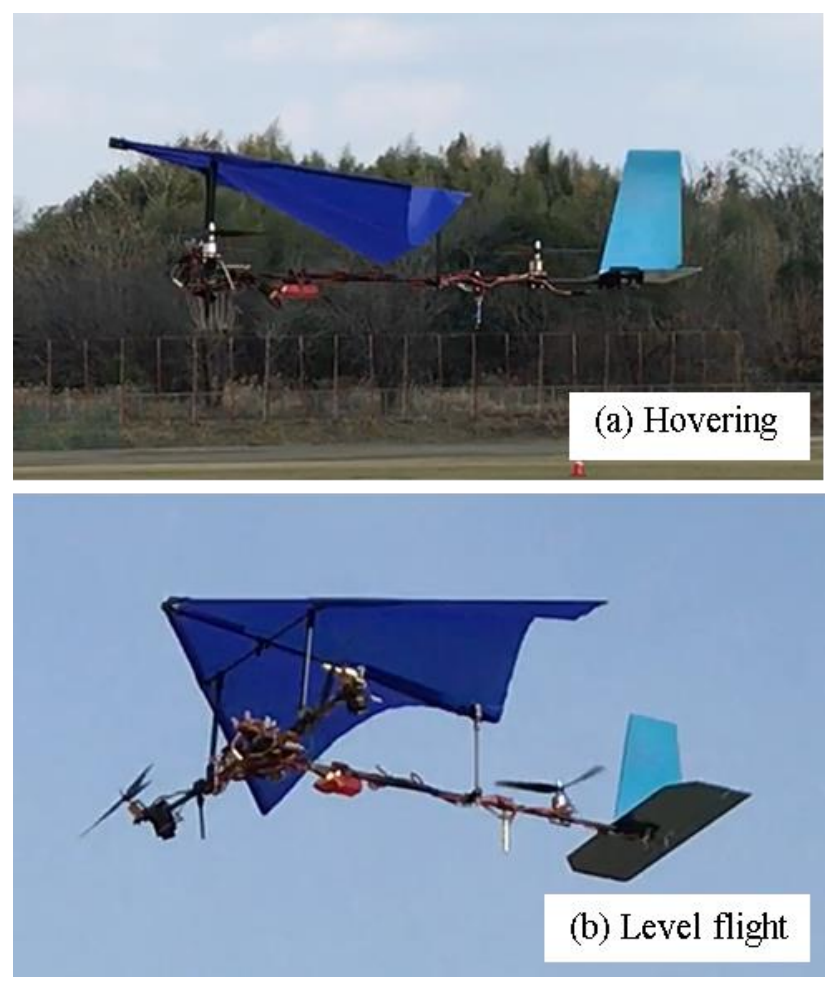

Fig. 8. Flight image of the proposed aircraft.

In order to evaluate the power saving performance of the aircraft, the test flight had done with logging the flight parameters. Table 1 shows the result of typical power consumption calculated from the flight logs of battery voltage and current. The power consumption of well-known commercial fixed-wing aircraft (Parrot Disco) of similar size is also listed in the table. Compared with the hovering, the aircraft during level flight can fly with less power by the lift of kite wing. The aircraft was able to fly with about $83 \%$ electric power at $45^{\circ}$ tilted rotors, and about $69 \%$ at $90^{\circ}$ tilted rotors.

For example, drone delivery to remote areas, fixed wing aircraft is advantageous of the flight efficiency, but there is difficulty in take-off and landing. We believe that proposed aircraft is useful for drone delivery to middle range areas that becomes difficult for normal drone. The aircraft is also advantageous for observing dangerous parts that cannot enter surroundings like volcanoes.

Table 1. Power consumption of proposed aircraft at difference angle of front rotors and fixed wing aircraft

\begin{tabular}{|c|c|}
\hline Flight attitude & Power consumption \\
\hline Hovering without tilt of rotors & $93 \mathrm{~W}$ \\
\hline Level flight with $45^{\circ}$ Tilt rotors & $77 \mathrm{~W}$ \\
\hline Level flight with $90^{\circ}$ Tilt rotors & $64 \mathrm{~W}$ \\
\hline Fixed wing aircraft (Parrot Disco) & $40 \mathrm{~W}$ \\
\hline
\end{tabular}

\section{Conclusions}

The trial production of a new concept VTOL aircraft of kite wing attached multicopter with tilt front rotos was carried out. Continuous transition from hovering to level flight can be done by tilting the front rotors. The lift of kite wing during horizontal flight supported the weight of the aircraft, and its causes the reduction of power consumption. More detailed flight data and the advantages for the wide range observation will be demonstrated in near future.

\section{Acknowledgment}

This work is partially supported by Grant-in-Aid for Scientific Research (C) 17K06952.

\section{References}

(1) Jan Wendel, Oliver Meister, Christian Schlaile and Gert F. Trommer, "An integrated GPS/MEMS-IMU navigation system for an autonomous helicopter", Aerospace Science and Technology, Vol.10, Issue 6, 2006, pp.527-533.

(2) Koichi Kita, Atsushi Konno, and Masaru Uchiyama," Hovering Control of a Tail-Sitter VTOL Aerial Robot", Journal of Robotics and Mechatronics, Vol.21 No.2 pp. 277-283.

(3) Ryan X-13 Vertijet, (accessed 26 may 2017), https://en.wikipedia.org/wiki/Ryan_X-13 Vertijet

(4) Jeremiah Gertler, "V-22 Osprey Tilt-Rotor Aircraft: Background and Issues for Congress", March 10, 2011, Congressional Research Service Reports, (accessed may 26, 2017), http://www.fas.org/sgp/crs/weapons/ $\underline{\text { RL31384.pdf }}$

(5) Kiyoteru Hayama, Hirofumi Ohtsuka and Hiroki Irie, "Trial Production of Variable Pitch Wing Attached Multicopter for Power Saving and Long Flight", Proc. of the 5th IIAE Int. Conf. on Intelligent Systems and Image Processing 2017 (ICISIP 2017), pp.36-41.

(6) Kiyoteru Hayama, Hiroki Irie, "Trial Production of Kite Wing Attached Multicopter for Power Saving and Long Flight", ICIC Express Letters, Part B: Applications, Vol. 10, Issue 5, May 2019, pp.405-412.

(7) CC Hardware Configuration-LibrePilot Documentation -Confluence,https://librepilot.atlassian.net/wiki/spaces/ LPDOC/pages/2818090/ (accessed June 6, 2019).

(8) LibrePilot-Open-Collavorative-Free,https://www.librep ilot.org/site/index.html, (accessed June 6, 2019). 